\title{
Coronary microvascular dysfunction: a great challenge for Cardiology
}

\author{
Claudio Pascale \\ Internal Medicine, Cottolengo Hospital, Torino, Italy
}

One of the main achievements of modern Cardiology has certainly been the possibility of visualizing and studying epicardial coronary arteries through selective angiography and consequently of evaluating the presence of more or less significant stenoses. ${ }^{1}$ Moreover, it is possible to find some acute obstructions, due to fissuring and disruption of an atherosclerotic unstable plaque and the production of an obstructive thrombus. ${ }^{2}$ The introduction of increasingly sophisticated methods has given the possibility of reopening critical coronary stenoses in order to achieve reperfusion of the ischemic myocardial areas.

Also the coronary artery spasm, an intense and acute vasoconstriction of an epicardial coronary artery that causes vessel narrowing or occlusion, has been described and the complex pathophysiological aspects and the clinical relevance have been studied. ${ }^{3}$

Revascularization for stable coronary artery disease by either percutaneous coronary intervention (PCI) or coronary artery bypass graft (CABG) is indicated in patients with persistence of symptoms despite medical treatment and/or in order to improve the prognosis in

Correspondence: Claudio Pascale, Internal Medicine, Cottolengo Hospital, via Cottolengo 9, 10152 Torino, Italy.

Tel.: +39.011.5294435 - Fax: +39.011.5294301.

E-mail: claudiopascale@ospedalecottolengo.it

Key words: Coronary microvascular dysfunction; coronary microvascular obstruction; coronary artery disease; ST-elevation myocardial infarction.

Conflict of interest: the author declares no potential conflict of interest.

Received for publication: 23 September 2015.

Accepted for publication: 23 September 2015.

This work is licensed under a Creative Commons Attribution NonCommercial 4.0 License (CC BY-NC 4.0).

(C) Copyright C. Pascale, 2016

Licensee PAGEPress, Italy

Italian Journal of Medicine 2016; 10:1-3

doi:10.4081/itjm.2016.665 flow-limiting coronary stenoses, by reducing myocardial ischemia and its adverse clinical manifestations. ${ }^{4}$ As regards the ST-elevation myocardial infarction (STEMI), caused in most cases by acute thrombotic obstruction of a coronary artery, primary PCI is the recommended method of reperfusion when it can be promptly performed by experienced operators. ${ }^{5}$ Thus the substantial improvement of medical care (which includes optimal pharmacological therapy and early revascularization) significantly decreased in-hospital mortality (approximately 5\% to 6\%) and 1-year mortality (approximately $7 \%$ to $18 \%$ ) from STEMI. ${ }^{6,7}$

But, almost paradoxically, while it is relatively easy to study and, if necessary, to reopen epicardial coronary arteries, the pathophysiological problem of coronary ischemia in its various clinical aspects cannot absolutely be explained only at this level. The clinical practice has clearly demonstrated that ischemia and angina cannot always be cured by removing a plaque which causes critical coronary stenosis. Some patients with angina pectoris do not have visible coronary atherosclerosis at angiography. In other patients, treated by PCI, myocardial ischemia persists despite stenosis removal. Many of these cases can be explained by a coronary microvascular dysfunction (CMD). ${ }^{8} \mathrm{~A}$ dysfunction of the coronary pre-arterioles (from 100 to $500 \mathrm{~mm}$ in diameter) and of the small coronary intramural arterioles $(<100 \mathrm{~mm}$ in diameter) can underlie microvascular angina. ${ }^{9}$ Coronary flow reserve may be impaired, in the absence of epicardial artery obstructions, by many and complex pathophysiological mechanisms: non-homogeneous metabolic vasodilation that may favor the steal phenomenon, inappropriate pre-arteriolar/arteriolar vasoconstriction, or other alterations. ${ }^{10}$ The diagnosis (electrical, mechanical, perfusive) of myocardial ischemia in CMD is most difficult, also for the peculiar distribution of ischemia. In the case of an epicardial coronary stenosis, myocardial ischemia diffusely involves the whole myocardial territory supplied by the vessel, in a homogeneous way and widely enough to cause regional perfusive and contractile dysfunction. In the case of coronary microvascular alterations, ischemic areas are patchily present in areas with normal myocardium. For this rea- 
son the detection of possible contractile abnormalities is very difficult. ${ }^{10}$

The recent European Guidelines on the management of stable coronary artery disease ${ }^{11}$ emphasize that there are different underlying mechanisms of stable coronary artery disease, among which the microvascular dysfunction, clinically characterized by typical chest pain, abnormalities of the electrocardiogram and/or stress test indicative of myocardial ischemia, but without fixed or dynamic obstructions in epicardial coronary arteries at coronary angiography. Some studies have confirmed a relatively higher mortality and worse outcome for patients with microvascular angina, which was formerly considered at low risk. ${ }^{12}$

Coronary microvascular dysfunction may also have acute forms of clinical presentation as unstable angina, or may be involved in apical ballooning syndrome (Takotsubo syndrome). ${ }^{8}$

Camici and Crea proposed a new and very interesting classification of CMD, both clinical and pathogenetic. ${ }^{9} \mathrm{CMD}$ was classified into four main types: i) $\mathrm{CMD}$ in the absence of myocardial diseases and obstructive coronary artery disease; ii) CMD in myocardial diseases (hypertrophic cardiomyopathy, dilated cardiomyopathy, myocarditis, aortic stenosis); iii) CMD in obstructive coronary artery disease (stable angina, acute coronary syndrome); iv) iatrogenic CMD (PCI, CABG).

The remarkable review published in this Journal by a group of some of the most important researchers in this field analyses a particular aspect of microvascular coronary dysfunction, connected to the recanalization in STEMI of the infarct-related artery by urgent PCI treatment or fibrinolysis. As said above, primary PCI is the elective treatment for STEMI, if promptly performed. But, in spite of the fact that the so-called door-to-balloon time has been significantly reduced in the past 10 years, in a sizable number of patients primary PCI achieves epicardial coronary artery recanalization but does not achieve myocardial reperfusion. So, in the last few years, in-hospital mortality from STEMI has remained virtually unchanged. ${ }^{13}$ The cause is to be found in the coronary microvascular obstruction (CMVO), which cancels the benefit obtained by the reopening of the obstructed coronary, failing to reduce the infarct side. CMVO is associated with an increased prevalence of early postinfarction complications, adverse left ventricular remodeling, late heart failure and death. ${ }^{14}$

Four interacting mechanisms are the cause of CMVO: distal embolization, ischemia-related injury, reperfusion-related injury and individual susceptibility of the microcirculation to injury. ${ }^{15}$ In the review by Scalone, Niccoli and Crea the mechanisms, invasive and non-invasive diagnoses and treatment strategies of CMVO are discussed in depth. ${ }^{16}$ The critical eval- uation of the possible interventions divided into different time windows, before-, in-, after-catheterization laboratory, is particularly notable.

As doctor Crea wrote in a recent NEJM editorial: Preexisting coronary microvascular dysfunction is emerging as a causal factor for future acute coronary events and for a worse response to reperfusion therapies. Thus, an integrated and personalized approach addressing all mechanisms in different time windows is needed in order to reduce the strikingly increased risk conferred by coronary microvascular obstruction. ${ }^{15}$

Physicians, particularly cardiologists and internists, should realize the importance of coronary microvascular dysfunction in the pathogenesis of myocardial ischemia, in the absence or in the presence of myocardial diseases and/or obstructive coronary artery disease. In the treatment of both acute and chronic myocardial ischemia, after the results achieved through the reopening of obstructed epicardial coronary arteries, the prevention and the therapy of coronary microvascular dysfunction seem to be one of the greatest challenges.

\section{References}

1. Gould KL, Lipscomb K. Effects of coronary stenoses on coronary flow reserve and resistance. Am J Cardiol 1974;34:48-55.

2. Depre C, Wijns W, Robert AM, et al. Pathology of unstable plaque: correlation with the clinical severity of acute coronary syndromes. J Am Coll Cardiol 1997;30: 694-702.

3. Lanza GA, Careri G, Crea F. Mechanisms of coronary artery spasm. Circulation 2011;124:1774-82.

4. Windecker S, Kolh P, Alfonso F, et al. 2014 ESC/EACTS Guidelines on myocardial revascularization: the Task Force on Myocardial Revascularization of the European Society of Cardiology (ESC) and the European Association for Cardio-Thoracic Surgery (EACTS) developed with the special contribution of the European Association of Percutaneous Cardiovascular Interventions (EAPCI). Eur Heart J 2014;35:2541-619.

5. O'Gara PT, Kushner FG, Ascheim DD, et al. 2013 ACCF/AHA guideline for the management of ST-elevation myocardial infarction: a report of the American College of Cardiology Foundation/American Heart Association Task Force on Practice Guidelines. Circulation 2013;127:e362-425.

6. Roe MT, Messenger JC, Weintraub WS, et al. Treatments, trends, and outcomes of acute myocardial infarction and percutaneous coronary intervention. J Am Coll Cardiol 2010;56:254-63.

7. Jernberg T, Johanson P, Held C, et al. Association between adoption of evidence-based treatment and survival for patients with ST-elevation myocardial infarction. JAMA 2011;305:1677-84.

8. Crea F, Camici PG, Bairey Merz CN. Coronary microvascular dysfunction: an update. Eur Heart J 2014; 35:1101-11. 
9. Camici PG, Crea F. Coronary microvascular dysfunction. N Engl J Med 2007;356:830-40.

10. Lanza GA, Crea F. Primary coronary microvascular dysfunction: clinical presentation, pathophysiology, and management. Circulation 2010;121:2317-25.

11. Task Force Members, Montalescot G, Sechtem U, et al. 2013 ESC guidelines on the management of stable coronary artery disease: the Task Force on the management of stable coronary artery disease of the European Society of Cardiology. Eur Heart J 2013;34:2949-3003.

12. Jespersen L, Hvelplund A, Abildstrom SZ, et al. Stable angina pectoris with no obstructive coronary artery disease is associated with increased risks of major adverse cardiovascular events. Eur Heart J 2012;33:734-44.
13. Menees DS, Peterson ED, Wang Y, et al. Door-to-balloon time and mortality among patients undergoing primary PCI. N Engl J Med 2013;369:901-9.

14. Bolognese L, Carrabba N, Parodi G, et al. Impact of microvascular dysfunction on left ventricular remodeling and long-term clinical outcome after primary coronary angioplasty for acute myocardial infarction. Circulation 2004;109:1121-6.

15. Crea F. Coronary microvascular obstruction - a puzzle with many pieces. N Engl J Med 2015;372:1464-5.

16. Scalone G, Niccoli G, Crea F. Pathophysiological aspects and management workflow of coronary microvascular obstruction in ST segment elevation myocardial infarction. Ital J Med 2016;10:10-22. 